\title{
Role of CT/MRI in Diagnosis of Gallbladder Malignancy
}

\author{
Naveen Sheelavant ${ }^{1}$, Ravi Kumar Yeli ${ }^{1}$, Naga Babu Pyadala ${ }^{2}$ \\ ${ }^{1}$ Assistant Professor, Department of Radiology \& Imaging, B M Patil Medical Collage \& Research Centre, BLDE University \\ (DU), Vijayapur, Karnataka, ${ }^{2}$ Associate Professor, Department of Biochemistry, MNR Medical College \& Hospital, Sangareddy, \\ Telangana, India
}

Corresponding author: Dr. Ravi Kumar Yeli, Assistant Professor, Department of Radiology \& Imaging, Shri B M Patil Medical Collage \& Research Centre, BLDE University (DU), Ashram Road, Vijayapur, Karnataka - 586103, India

DOI: http://dx.doi.org/10.21276/ijcmsr.2020.5.2.8

How to cite this article: Naveen Sheelavant, Ravi Kumar Yeli, Naga Babu Pyadala. Role of CT/MRI in Diagnosis of gallbladder malignancy. International Journal of Contemporary Medicine Surgery and Radiology. 2020;5(2):B34B37.

\section{A B S T R A C T}

Introduction: Gallbladder cancer carries an extremely high mortality rate, with a 5-year survival rate as low as $12 \%$. Survival is dependent on the diagnosis of these tumors in their earliest stages. This study sought to describe the clinical and imaging features of stages T1, T2, and T3 gallbladder tumors and to illustrate features that may allow radiologists to make an early diagnosis.

Material and Methods: After approval from the institutional review board, a search of the pathology department database yielded 16 patients with surgically proven T1, T2, and T3 gallbladder cancers with available preoperative computed tomography (CT) or magnetic resonance imaging (MRI). The imaging was reviewed for lesional morphology (focal polyploid mass, focal wall thickening, circumferential wall thickening), enhancement characteristics, liver invasion, locoregional lymphadenopathy, and distant metastatic disease. The electronic medical record was also searched for demographic information and clinical presentation.

Results: There was 9 women and 7 men with a mean age of 67 years. Virtually all patients were symptomatic, with most patients demonstrating symptoms suggestive of underlying malignancy (including jaundice, weight loss, and chronic abdominal pain). Tumors on CT and MRI included 6 polyploid masses, 7 tumors with focal wall thickening, and 3 with circumferential wall thickening.

Conclusion: The imaging findings of gallbladder cancer can be subtle, regardless of whether the tumor presents as a discrete mass, focal wall thickening, or circumferential diffuse wall thickening, and radio-logists should be aware of the wide range of different possible appearances. Moreover, the vast majority of these patients had clinical symptoms suggestive of an underlying malignancy, and this should precipitate a careful evaluation of the gallbladder in all such cases.

Keywords: Gallbladder Carcinoma, Computed Tomography, Magnetic Resonance Imaging.

\section{INTRODUCTION}

Although a relatively rare malignancy, gallbladder cancer is the fifth most common tumor of the gastrointestinal tract and still accounts for more than $60 \%$ of all biliary tract malignancies, with more than 6000 new cases diagnosed in the United States in 2012. ${ }^{1-3}$ Gallbladder carcinoma carries an extraordinarily poor prognosis, even in those patients who undergo surgical resection with a curative intent, with 5-year survival rates as low as $12 \% .{ }^{4}$ This poor prognosis stresses the importance of identifying these carcinomas in their earliest stages because patients with T1 (tumors confined to the lamina propria or muscular layer of the gallbladder wall) and T2 (tumors extending into the muscularis mucosa) lesions have a much better prognosis compared with patients with T3 or T4 lesions (tumors no longer confined to the gallbladder). ${ }^{5-7}$ Tumors that are found in their earliest stages can often be treated with a simple or extended cholecystectomy, and patients have a better chance for longterm survival. On the other hand, only $10 \%$ to $30 \%$ of patients with advanced stage gallbladder cancer are candidates for curative resection, and those patients who are still surgical candidates require much more extensive surgical resections. ${ }^{2}$ Unfortunately, the prospective identification of these lesions using cross-sectional imaging techniques remains very difficult, and many of these tumors are incidentally found either on autopsy or during cholecystectomy for another indication. ${ }^{8}$ In many cases of gallbladder cancer, the imaging findings can be extraordinarily subtle and difficult to perceive for the radiologist, and prospective identification of these malignancies requires an understanding of the most common appearances and clinical presentations of these lesions while still in their earliest stages. As a result, this study sought to retrospectively evaluate the imaging appearance on computed tomography (CT) and magnetic resonance imaging (MRI) of patients with T1, T2, and T3 gallbladder cancers.

\section{MATERIAL AND METHODS}

Our institutional review board approved the study, and we 
received a waiver of informed consent for review of both clinical records and any prior imaging examinations. A search of the pathology department database was then conducted, yielding 16 patients with pathologically proven T1, T2, or T3 gallbladder cancer and with imaging available on picture archiving and communication system (PACS) at our institution (either CT or MRI). Patients with T4 gallbladder cancers were excluded from the study. Once the patients were identified, the electronic medical record was then reviewed for clinical and demographic information, including patient age, sex, presenting symptoms, and the like. In addition to retrospective $\mathrm{CT}$ and $\mathrm{MRI}$ review, the original dictations for

\begin{tabular}{|l|c|}
\hline Category & Total numbers \\
\hline Men & 7 \\
\hline Women & 9 \\
\hline Mean age & 67 years \\
\hline Weight loss & 2 \\
\hline Chronic abdominal pain & 3 \\
\hline Acute abdominal pain & 2 \\
\hline Jaundice & 10 \\
\hline Nausea and vomiting & 1 \\
\hline None & 1 \\
\hline Unknown symptoms & 1 \\
\hline
\end{tabular}

Table-1: Demographic and Clinical Information of Gallbladder Cancer patients $(n=16)$.

\begin{tabular}{|l|c|}
\hline Category & Total \\
\hline T1 & 2 \\
\hline T2 & 4 \\
\hline T3 & 10 \\
\hline T4 & 0 \\
\hline N0 & 2 \\
\hline N1 & 8 \\
\hline M0 & 14 \\
\hline Mx & 2 \\
\hline Time between imaging and surgery mean & $0-0.071$ \\
\hline $\begin{array}{l}\text { No. patients without distant metastatic disease at } \\
\text { the last follow-up }\end{array}$ & 9 \\
\hline $\begin{array}{l}\text { No. patients who developed metastatic disease } \\
\text { after surgery }\end{array}$ & 3 \\
\hline \multicolumn{2}{|c|}{ Table-2: Pathologic findings of gallbladder cancer patients } \\
(n=16).
\end{tabular}

each of the patients' preoperative imaging examinations were also reviewed to ascertain whether the patients' gallbladder cancers were diagnosed prospectively (including CT, MRI, and ultrasound). The available CT and MRI examinations (16 patients with only CT, 2 patients with only MRI) for each patient were interpreted by a board-certified radiologist with subspecialty training in abdominal imaging and 2 years of experience after fellowship. Each of the studies (regardless of the imaging modality) was interpreted with regard to the following features: (a) presence of gallstones, (b) presence of focal gallbladder thickening (including the length and thickness of that abnormality), (c) presence of diffuse gallbladder wall thickening (including measurement of thickness), (d) presence of a focal mass within the gallbladder (including size of that mass), (e) Hounsfield attenuation measurements in any available phases of imaging (on CT), (f) presence of suspicious locoregional lymphadenopathy, (g) assessment of possible liver invasion, (h) presence of peritoneal or omental implants/carcinomatosis, and (i) evidence of superimposed cholecystitis.

\section{RESULTS}

Patient demographics (age, sex) and presenting symptoms are listed in Table 1 . The patients were relatively equally divided by sex ( 9 women, 7 men), and the mean age at presentation was 67 years. Of the 15 patients for whom preoperative notes were available, the most common presenting symptom was jaundice (10 patients). Whereas most of the patients had symptoms at least suggestive of malignancy, 2 patients presented only with acute abdominal pain. Pathologic staging information, the time between surgery and imaging, and follow-up information are listed in Table 2. Notably, 2 of the 4 patients who were initially found to have distant metastatic disease at presentation had their metastases (carcinomatosis) discovered only at surgery. Three of these 4 patients had T3 tumors (1 was T2). Four patients later developed distant metastatic disease, all of whom had T3 tumors. Five patients had evidence at pathology of direct tumor invasion into the liver, 3 of which were correctly identified on preoperative CT. Three additional patients were incorrectly thought to have liver invasion on the basis of CT. The MDCT imaging data are presented in Table 3. 4 patients presented with a discrete polyploid mass, 9 patients presented with focal wall thickening, and 3 patients presented with diffuse circumferential wall thickening. Seven patients were thought

\begin{tabular}{|l|c|}
\hline Category & Total \\
\hline Polyploid mass & 4 \\
\hline Focal wall thickening & 9 \\
\hline Diffuse circumferential wall thickening & 3 \\
\hline Size of polyploid masses & $15-61 \mathrm{~mm}$ \\
\hline Wall thickness for tumors presenting as circumferential wall thickening, mean/median (range) & $9-23 \mathrm{~mm}$ \\
\hline Wall thickness for tumors presenting as focal wall thickening, mean/median (range) & $8-24 \mathrm{~mm}$ \\
\hline Length of thickening for tumors presenting as focal wall thickening, mean/median (range) & $13-80 \mathrm{~mm}$ \\
\hline Hounsfield attenuation tumor arterial phase & $25-108$ \\
\hline Hounsfield attenuation tumor venous phase & $33-219$ \\
\hline Change in attenuation (venous-arterial) & $5-129$ \\
\hline \multicolumn{2}{|c|}{ Table-3: Imaging Findings for the 16 Patients with Preoperative CT or MRI. } \\
\hline
\end{tabular}


to have suspicious locoregional lymphadenopathy (based on size, morphology, or central necrosis): 4 of these patients were found to have malignant lymphadenopathy at lymph node dissection, whereas the other 3 patients did not undergo lymph node sampling at surgery. Two patients were correctly identified as having distant metastatic disease on preoperative imaging (1 with carcinomatosis, 1 with liver metastases), whereas carcinomatosis found at surgery was not perceived on 2 other CT studies. Four patients were found to have gallstones on CT. Enhancement increased from the arterial to venous phases in all cases with dual-phase imaging. The mean attenuation of those tumors imaged with CT was 59.4 on the arterial phase and 86.5 on the venous phase, with a mean increase in Hounsfield attenuation between the arterial and venous phases of 28.2 HUs.

\section{DISCUSSION}

Gallbladder cancer carries a dismal prognosis, with an overall survival rate reported as low as $12 \% .{ }^{4,5}$ Virtually, all survivors of this tumor harbor a lower stage of disease, and patients with more advanced tumors (higher stage) have extremely low survival rates. ${ }^{5}$ The $\mathrm{T}$ stage of these tumors carries great significance in terms of both prognosis and treatment: T1 lesions, which are confined to the lamina propria or the muscular layer of the gallbladder wall, can usually be treated adequately with a simple laparoscopic cholecystectomy. ${ }^{7} \mathrm{~T} 2$ tumors, which have grown into the perimuscular fibrous tissue/muscularis mucosa, are typically treated with extended cholecystectomy or even more extensive surgeries. ${ }^{9}$ T3 (tumors that have grown into the serosa and/or directly into either the liver or other adjacent structures) and T4 (tumors with vascular involvement, more than $2 \mathrm{~cm}$ of invasion into the liver, or involvement of 2 or more structures other than the liver) tumors carry an extremely poor prognosis, and although many of these tumors may be unresectable, radical resection is necessary in those cases with a potentially resectable tumor. Although our study population was small, it generally supported the poor prognosis of these tumors, with 4 and 9 patients (of 16) demonstrating metastatic disease and malignant locoregional lymphadenopathy at presentation, respectively, and an additional 4 patients developing progressive metastatic disease during the course of their follow-up. Notably, however, of the 7 patients with T1 and T2 tumors, only 1 demonstrated distant metastatic disease and 3 demonstrated locoregional lymphadenopathy at presentation (and none during the follow-up), underscoring the importance of identifying these tumors early in their course. It is important to recognize that, in the vast majority of these cases, the patient's clinical presentation should have alerted the radiologist to be vigilant about the presence of malignancy: The most common clinical presentations included jaundice, weight loss, and chronic abdominal pain, all histories suggestive of underlying malignancy. Despite this, 2 patients presented with acute abdominal pain and 1 patient had no appreciable symptoms, such that the absence of an appropriate history cannot necessarily exclude the presence of a cancer. The accuracy of CT in the diagnosis of gallbladder cancer has previously been reported as $84 \%$ to $92 \%$, with sensitivities and specificities ranging from $73 \%$ to
$87 \%$ and $88 \%$ to $100 \%$, respectively. ${ }^{10-12}$ Features that have been previously described as suggestive of a gallbladder malignancy on CT include a discrete focal gallbladder mass, irregular focal wall thickening, and a "2-layer pattern" of enhancement in a thickened gallbladder wall (with a weakly enhancing outer layer and a strongly enhancing, thickened inner layer). ${ }^{11,13}$ On the other hand, the performance of MRI in the diagnosis of gallbladder cancer has not been well established, although suggestive features are similar to those on CT, including a focal mass, focal gallbladder wall thickening, abnormal enhancement, and restricted diffusion (low apparent diffusion coefficient values).9,14,15 The results of this study support a wide range of different possible imaging appearances for gallbladder cancer, with 4 polyploid masses, 9 cases with focal wall thickening, and 3 cases with diffuse circumferential wall thickening. Interestingly, given the large number of other potential causes for diffuse circumferential wall thickening, including cholecystitis, adenomyomatosis, intrinsic liver disease, congestive heart failure, and renal failure, diffuse wall thickening has traditionally not thought to be suggestive of malignancy. ${ }^{16,17}$ Of note, in each of the 3 cases in this study that presented with diffuse wall thickening, there was a strong clinical history suggestive of malignancy (ie, jaundice, weight loss) that should have pointed toward the correct diagnosis. In each of these cases, the wall was substantially thickened up to an average of $14 \mathrm{~mm}$. Of the 16 patients in this series, 4 were incorrectly diagnosed prospectively, including 1 patient with a discrete polyploid mass (diagnosed with acute cholecystitis), 4 with focal wall thickening, and 1 with diffuse circumferential wall thickening. ${ }^{18}$ Most of the polyploid masses in this series were not difficult to diagnose, with a mean size of $37 \mathrm{~mm}$. However, the one case that was not prospectively identified was a small $\mathrm{T} 1$ lesion in a patient with acute abdominal pain, no symptoms suggestive of malignancy, and a presumptive diagnosis of cholecystitis. By far, the most common appearance of gallbladder cancer in this series was focal wall thickening (in 9 of the 16 patients), which also accounted for most incorrect prospective diagnoses. Viewed as a group, these tumors were associated with substantial focal wall thickening, extending over an average length of $32.7 \mathrm{~mm}$ with an average thickness of $12.2 \mathrm{~mm}$. All 4 of these incorrectly diagnosed cases with focal wall thickening occurred in patients with clinical symptoms of jaundice, and in 3 of the 4 cases, gallbladder thickening was noted, but the focality of this thickening was not appropriately recognized as cancer. Clearly, to arrive at the correct diagnosis, patient symptomatology must be strongly taken into account; moreover, when wall thickening is identified, the thickened wall must be more carefully examined to distinguish a diffusely thickened wall from focal thickening. Nevertheless, given the patients in this series who presented with either no symptoms or symptoms of acute abdominal pain, clinical presentation alone should not be relied upon solely to guide a radiologist's search pattern or to arrive at the correct diagnosis. From the perspective of tumor enhancement, there was a significant variability in the appearance of these tumors in both the arterial and venous phases. Every tumor in this series with dual-phase imaging showed a greater degree of 
enhancement on the venous phase images compared with those of the arterial phase, with a mean difference in Hounsfield attenuation between the 2 phases of $28.2 \mathrm{HU}$. Despite this, there was a dramatic variation in the degree of enhancement between different tumors: A few tumors showed substantial vascularity (HU more than 90 on both the arterial and venous phase images), whereas most cases showed attenuations under $90 \mathrm{HU}$ on both phases. There is little to suggest that the degree of enhancement on either phase of imaging can help make the diagnosis of a malignancy, as opposed to an inflammatory process, and there was certainly no consistent pattern of enhancement across the cases in our series. There are several limitations of our study that should noted, including the relatively small sample size. The vast majority of gallbladder cancers identified at any institution tend to be unresectable $\mathrm{T} 4$ tumors with metastatic disease or bulky locoregional lymphadenopathy. As a result, our study size is small; moreover, most lesions are T3 tumors, with only a few gallbladder-confined T1 and T2 lesions. Nevertheless, given that some T3 tumors are still potentially resectable, these cases stress the critical need for radiologists to identify these T3 tumors while they are still potentially in a resectable state. Second, very few of our patients underwent MRI, limiting our ability to make any definitive statements about the MRI appearance of these tumors. As a result, we have refrained from drawing any strong conclusions about the role of MRI in the evaluation of gallbladder malignancies. Finally, given that not all of our patients underwent dualphase CT imaging, it would be interesting to look at a larger number of these tumors and gain a better sense of the true enhancement characteristics of these lesions. Gallbladder cancer is associated with extraordinary high mortality and poor clinical outcomes, making it imperative that radiologists correctly identify these lesions in their earliest stages. As the cases in this series illustrate, the imaging findings can be quite subtle, regardless of whether the tumor presents as a discrete mass, focal wall thickening, or circumferential diffuse wall thickening, and radiologists must be aware of the wide range of different possible appearances. Moreover, the vast majority of these patients had clinical symptoms suggestive of an underlying malignancy, and this should precipitate a careful evaluation of the gallbladder in all such cases.

\section{ACKNOWLEDGEMENT}

Authors are thankful to Director and Dean of B M Patil Medical Collage \& Research Centre, Vijayapur.

\section{REFERENCES}

1. Gallbladder Cancer. American Cancer Society. November 20, 2013. Available from: http:// www.cancer. org/cancer/gallbladdercancer/detailedguide/index.

2. Kalra N, Suri S, Gupta R, et al. MDCT in the staging of gallbladder carcinoma. AJR Am J Roentgenol. 2006; 186(1):758-762.

3. Deshmukh SD, Johnson PT, Sheth S, et al. CT of gallbladder cancer and its mimics: a pattern-based approach. Abdom Imaging. 2013; 38(6):527-536.

4. DS. Carcinoma of the gall-bladder: an experience and review of the literature. Aust N Z J Surg. 1995;
65(2):724-727.

5. Fong Y, Jarnagin W, Blumgart LH. Gallbladder cancer: comparison of patients presenting initially for definitive operation with those presenting after prior noncurative intervention. Ann Surg. 2000; 232(5):557-569.

6. Edge, S.; Byrd, D.; Compton, C., et al. AJCC Cancer Staging Manual. 7th ed. Edge, S.; Byrd, D.; Compton, C., et al., editors. Springer; New York, NY: 2010.

7. Kim SJ, Lee JM, Lee JY, et al. Accuracy of preoperative T-staging of gallbladder carcinoma using MDCT. AJR Am J Roentgenol. 2008; 190(3):74-80.

8. Polk HC Jr. Carcinoma and the calcified gall bladder. Gastroenterology. 1966; 50(4):582-585.

9. Yoshimitsu K, Nishihara Y, Okamoto D, et al. Magnetic resonance differentiation between $\mathrm{T} 2$ and $\mathrm{T} 1$ gallbladder carcinoma: significance of subserosal enhancement on the delayed phase dynamic study. Magn Reson Imaging. 2012; 30(1):854-859.

10. Furukawa H, Kosuge T, Shimada K, et al. Small polypoid lesions of the gallbladder: differential diagnosis and surgical indications by helical computed tomography. Arch Surg. 1998; 133(5):735- 739.

11. Kim SJ, Lee JM, Lee JY, et al. Analysis of enhancement pattern of flat gallbladder wall thickening on MDCT to differentiate gallbladder cancer from cholecystitis. AJR Am J Roentgenol. 2008; 191(3):765-771.

12. Tongdee R, Maroongroge $P$, Suthikeree W. The value of MDCT scans in differentiation between benign and malignant gallbladder wall thickening. J Med Assoc Thai. 2011; 94(2):592-600.

13. Yun EJ, Cho SG, Park S, et al. Gallbladder carcinoma and chronic cholecystitis: differentiation with twophase spiral CT. Abdom Imaging. 2004; 29(6):102-108.

14. Ogawa T, Horaguchi J, Fujita N, et al. High b-value diffusion-weighted magnetic resonance imaging for gallbladder lesions: differentiation between benignity and malignancy. J Gastroenterol. 2012; 47(4):13521360 .

15. Tan CH, Lim KS. MRI of gallbladder cancer. Diagn Interv Radiol. 2013; 19(5):312-319.

16. Ching BH, Yeh BM, Westphalen AC, et al. CT differentiation of adenomyomatosis and gallbladder cancer. AJR Am J Roentgenol. 2007; 189(1):62-66.

17. Uchiyama $\mathrm{K}$, Ozawa $\mathrm{S}$, Ueno $\mathrm{M}$, et al. Xanthogranulomatous cholecystitis: the use of preoperative CT findings to differentiate it from gallbladder carcinoma. J Hepatobiliary Pancreat Surg. 2009; 16(3):333-338.

18. Liang JL, Chen MC, Huang HY, et al. Gallbladder carcinoma manifesting as acute cholecystitis: clinical and computed tomographic features. Surgery. 2009; 146(5):861-868.

Source of Support: Nil; Conflict of Interest: None

Submitted: 19-03-2020; Accepted: 18-04-2020; Published online: 21-05-2020 